\title{
Enhanced Mobility Support for Roaming Users: Extending the IEEE 802.21 Information Service
}

\author{
Karl Andersson ${ }^{1}$, Andrea G. Forte ${ }^{2}$, and Henning Schulzrinne ${ }^{2}$ \\ ${ }^{1}$ Luleå University of Technology \\ Division of Mobile Networking and Computing \\ SE-931 87 Skellefteå, Sweden \\ karl.anderssonaltu.se \\ ${ }^{2}$ Columbia University \\ Department of Computer Science \\ New York, NY 10027, USA \\ \{andreaf, hgs\}@cs.columbia.edu
}

\begin{abstract}
Many cell-phones and Personal Digital Assistants (PDAs) are equipped with multiple radio interfaces. Because of this, devices need to have ways of efficiently selecting the most suitable access network across multiple technologies based on the physical location of the device as well as userdefined parameters such as cost, bandwidth, and battery consumption. The IEEE has standardized the 802.21 framework for media-independent handovers where dynamic selection of network interfaces is an important feature. This paper describes and evaluates a novel architecture which extends the IEEE 802.21 information service. The architecture is based on a three-layer structure with Location-to-Service Translation (LoST) servers, service provider servers and independent evaluator servers. Evaluator servers are populated with information on coverage and quality of service as provided by trusted users. The proposed architecture allows for competition at all levels and scales well due to its distributed nature. A prototype has been developed and is presented in the paper.
\end{abstract}

Keywords: Heterogeneous wireless environments, Media-independent handover services, IEEE 802.21 information service.

\section{Introduction}

In the near future, mobile users will likely have access to many different wireless access technologies, from 4G LTE to WiMAX, offering broad coverage, and hot spots offering IEEE 802.11 connectivity for smaller areas. Each of these networks offers a trade-off of cost, convenience and performance. For example, a user may decide to walk to a nearby hot spot rather than pay high per-MB cellular data rates to perform some data-intensive task. Also, the performance of the same kind of network differs by geographic location; data rates in dense metropolitan areas may be lower than outside those areas. However, currently, mobile users or their devices have no good way to make such choices without elaborate web searches and manual tariff comparisons. We propose a new model for automating the decision process that not only take 
into account static information, such as cost, but also allows to incorporate the experience real users have had with the network in that particular geographic area.

The rest of the paper is organized as follows. Section 2 presents an overview of mobility management schemes and the IEEE 802.21 standard while Section 3 presents the Location-to-Service Translation (LoST) protocol. Section 4 presents the proposed architecture while Section 5 evaluates the work by describing the prototype built, experiments being conducted, and results achieved. Finally, Section 6 contains related work, while Section 7 concludes the work and indicates future work.

\section{Mobility Management and the IEEE 802.21 Standard}

Seamless mobility is achieved by applying a suitable mobility management scheme handled at the link layer, the network layer [1, 2], the transport layer [3, 4], or the application layer [5]. Also, proposals on new layers for handling mobility exist [6] as well as proposals on handling mobility in the network [7], instead of using host-based solutions.

To improve handover performance in heterogeneous environments, the IEEE decided to standardize a media-independent handover (MIH) framework under the name of 802.21 [8]. It defines mechanisms for exchanging handover-related events, commands, and information. Handover initiation and handover preparation are covered but not the actual handover execution. It should also be noted that the mobility management mechanism can be of any type, working at either the network, transport or application layers. Finally, the IEEE 802.21 standard allows for both networkcontrolled handovers and host-controlled handovers and it defines three main services: Media-independent Event Services (MIES), Media-independent Command Services (MICS) and Media-independent Information Services (MIIS).

\subsection{Media-Independent Event Services (MIES)}

MIES define events representing changes in the link characteristics either originated from the link layer or from the MIH function. Such characteristics could be information on link status or link quality, for example. Events can be subscribed to and be either local or remote. They may indicate changes in the state and transmission behavior of the physical, data-link and logical-link layers. Events can also predict state changes of these layers. Remote events are transported over the network in MIH protocol messages and typically contain information on link events originated from the point of attachment that the user subscribed to earlier.

\subsection{Media-Independent Command Service (MICS)}

The MICS defines commands for controlling the link state and can be invoked either locally or remotely. By using the MICS, the user may control the configuration and selection of a specific link. Remote commands are, like remote events, transported over the network by MIH protocol messages and may result in a link command or an $\mathrm{MIH}$ indication in the peer Media-Independent Handover Function (MIHF) entity. 


\subsection{Media-Independent Information Service (MIIS)}

The MIIS defines a set of information elements (IEs), their structure and their representation. Furthermore, it defines a query-response-based mechanism for information retrieval. Such information can be used to take more accurate handover decisions that is, using information on available access networks in the proximity of the user may help to radically improve the decision-making process for handovers.

Information is exchanged through binary type-length-value (TLV) coded messages. Also, complex queries are supported through the Resource Description Framework (RDF) query language SPARQL [9].

IEs can be of general type indicating either the network type, operator identifier, or a service-provider identifier. They can also be access-network specific by providing specific information on Quality of Service (QoS), security characteristics, revisions of current technology standards in use, cost, and roaming partners. Also, some IE types deliver Point-of-Attachment (PoA)-specific information such as the MAC address of the PoA, its geographical location, data rates offered, and channel information. IEs may also be vendor-specific.

Figure 1 shows the MIH framework and communication between local and remote MIHF entities.

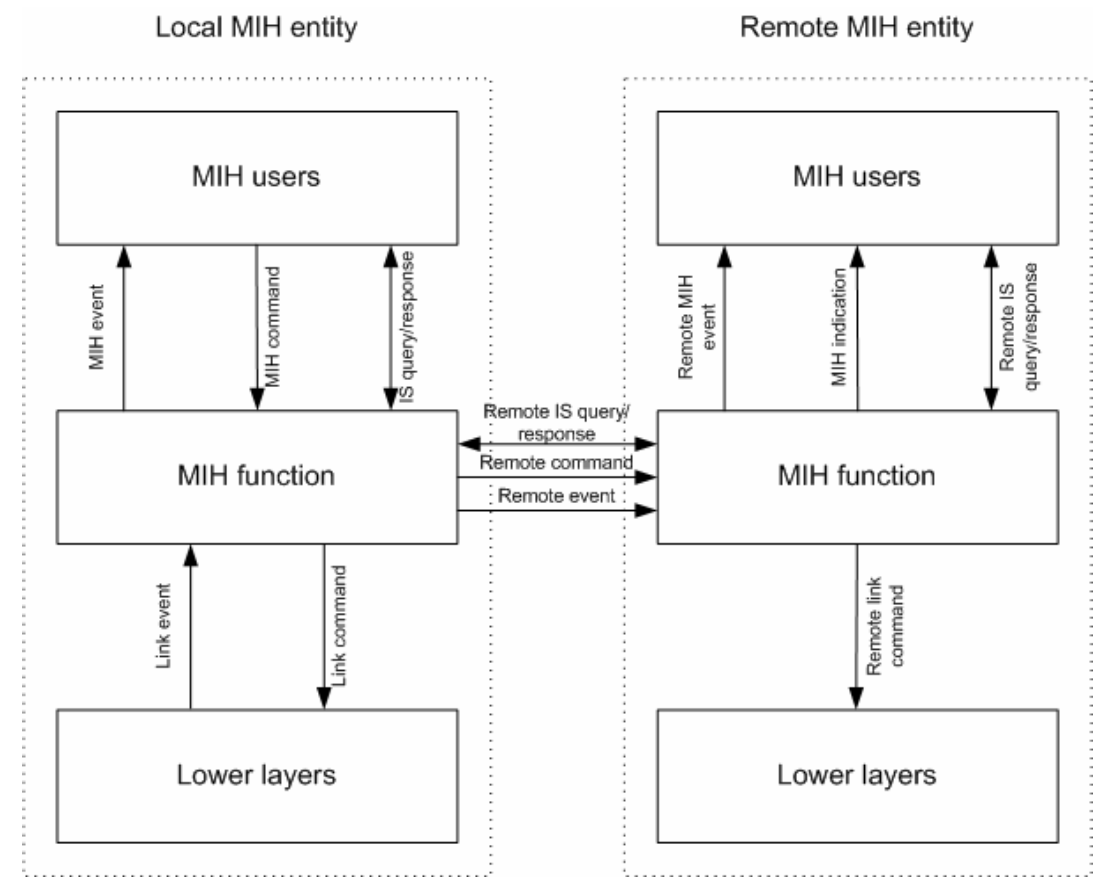

Fig. 1. Media-independent handover framework

The interfaces in the architecture are defined by a number of Service Access Points (SAPs) in the IEEE 802.21 standard. The interface between MIH users and the MIH function is referred to as the MIH_SAP while the interface to the lower layers is 
referred to as the MIH_LINK_SAP which is generic to all access technologies. The primitives in the MIH_LINK_SAP are mapped to technology-specific primitives included in the IEEE 802.21 standard. MIH_NET_SAP defines the exchange of messages between MIH entities.

MIH protocol messages are either sent at layer 2 or by using higher layer communication mechanisms [10, 11, 12, 13].

Currently, security extensions are being standardized (IEEE 802.21a), as well as handling of handovers for downlink only technologies (IEEE 802.21b).

\section{Overview of the Location-to-Service Translation (LoST) Protocol}

The Location-to-Service Translation (LoST) protocol [14] was originally developed to map location information into Uniform Resource Locators (URLs) representing Public Safety Answering Points (PSAPs) for emergency calling. Although targeted for a specific application, LoST offers great flexibility and is not at all limited to its initially targeted application. Therefore, a generalized Location-to-URL Mapping Architecture and Framework was also developed [15]. Furthermore, methods for finding LoST servers were described [16]. Ongoing work includes definition of LoST extensions [17], labels for common location-based services [18], and a policy for defining new service-identifying labels [19].

LoST uses a distributed architecture relaying heavily on caching. Protocol messages are carried in HTTP messages. The LoST architecture consists of seekers, resolvers, forest guides, and authoritative mapping servers (AMSs). Queries originate from seekers that want a location to be mapped into a URL. Resolvers are special servers with information on various jurisdictions and cached data typically operated by the Internet Service Provider (ISP) or an enterprise. Forest guides acts as a lightweight directory service for trees of AMSs, who in turn performs the mapping functionality, see Figure 2.

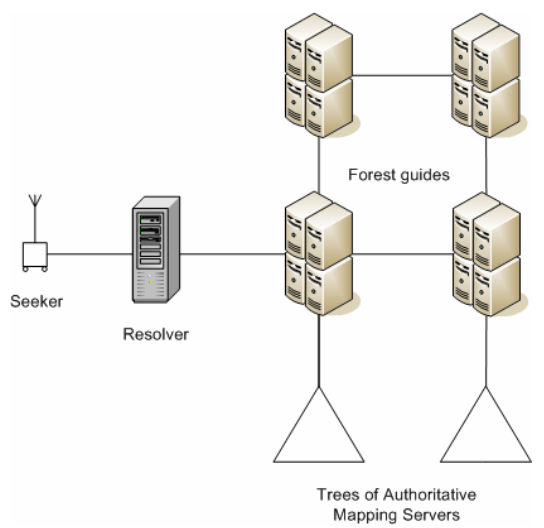

Fig. 2. Location-to-Service Translation (LoST) Architecture 
LoST is an XML-based protocol. The LoST client performing a query is called a seeker. Typically, a seeker sends a query to its resolver in order to find a mapping for the specified type of service, given the device physical location. If the resolver does not have such information it forwards the request one step up in the hierarchy to a forest guide. The forest guide knows the coverage area of all trees and can therefore propagate the query down to the correct tree. Eventually, in the tree the query will reach an authoritative server which will be able to send a reply. Such reply propagates back to the seeker by traversing the same servers traversed by the query but in reverse order. The path of the query is stored in $\langle\mathrm{via}\rangle$ elements allowing the response to follow the path in the opposite direction. There is also an iterative version of the query protocol allowing for servers to respond with addresses of other servers instead of actually delivering the answer to the query. This feature allows the seeker to iterate over a set of servers. The query $<$ findService $>$ is the core query type in LoST.

\section{Proposed Architecture}

The core contribution of this paper is the architecture proposal presented in this section. The architecture is built on a three-layer structure. At the top layer there are LoST top-level servers serving a specific geographic region. These servers perform location-to-service mapping to servers of the second and third layer. At the second layer service-provider-owned servers deliver information on each service provider's access network. Finally, at the third layer servers run by independent evaluators deliver aggregated information on available access networks that is, networks provided by national providers but also by local service providers (i.e., the local bookstore's WiFi network). Aggregated information is collected with users submitting reports which contain information on coverage and quality of service as they are experienced by users, see Figure 3.

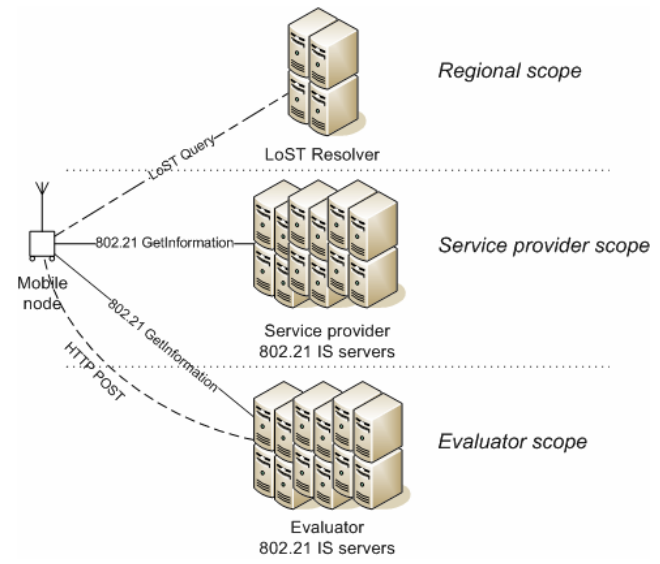

Fig. 3. Architecture proposal 
In a typical scenario the end node sends a LoST <findService $>$ query to the LoST resolver; such query containing current location of the end-user, a certain area within which the user wants to find points of service, and the 802.21 service URN. Figure 4 shows an example of a LoST <findService> geodetic query. In particular, with this query we are asking the LoST server to find IEEE 802.21 IS servers handling information about points of attachment within 200 meters from our current position.

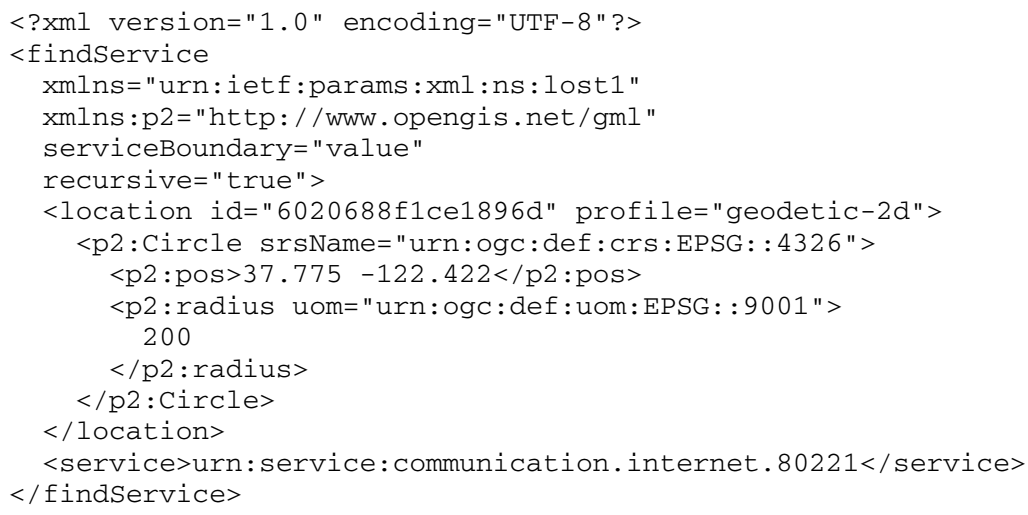

Fig. 4. LoST query example

The LoST server responds with a list of 802.21 IS servers corresponding to the service-provider 802.21 IS servers and evaluator-built 802.21 servers within the geographical area specified by the end-user in the query. After getting such list, the end node directly queries the chosen 802.21 IS server(s) in order to obtain a list of available networks and points of attachment. Also, end nodes may filter the list of points of attachment based on some criteria such as preferred network. At this point they would select the local interface to use and the network to connect to based on cost, battery consumption and performance. Required performance may change depending on the application to be used at a specific time.

For contributing information to evaluator-built 802.21 IS servers, selected identified users may submit reports at three levels. On one level such users can passively scan the medium and submit reports on points of attachment, including information such as MAC address/cell id, channels used, type of encryption used. On a second level, these selected users can submit extended reports for those access points or base stations they are actually connected to. This second type of reports would contain information such as achieved data rates, delay, jitter, or packet loss rate. Both types of reports include the location of the user and allow the server to estimate the location of the access points or base stations. The third and last type of report is the error report. Error reports are sent by clients to inform the 802.21 IS server that the received information was wrong. After receiving a certain number of error reports from different clients regarding the same access point or base station, the 802.21 IS server may decide to remove information regarding that access point or base station from its database. 
Reports are sent over HTTP-based POST messages to the 802.21 IS server which has been extended to handle this. The structure of these types of messages is following the IEEE 802.21 basic schema [20]. Figure 5 exemplifies an extended report.

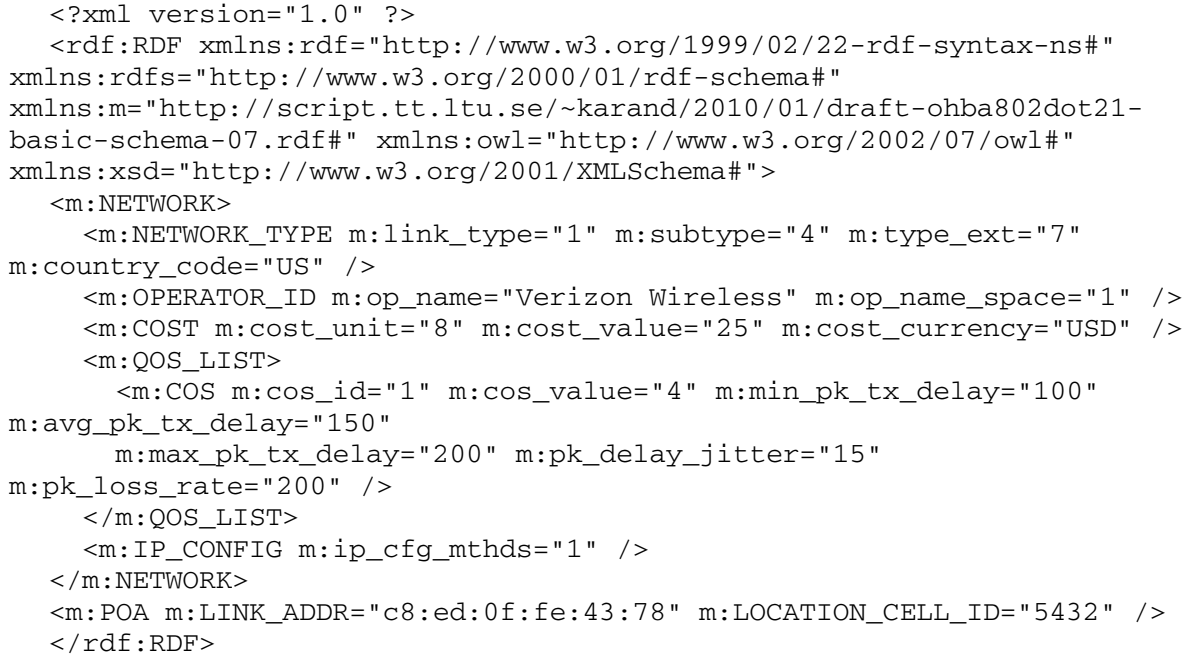

Fig. 5. Example report to an evaluator-built 802.21 IS server

By collecting such reports, an 802.21 IS server builds up its own independent database containing as rich data as any service-provider-owned 802.21 IS server could, while not being operator-network specific. Also, such independent 802.21 IS servers because of the way they are built and maintained, might be able to update their content faster than a manually-administered system.

Figure 6 shows messages exchanged between an end user and an evaluator-built 802.21 IS server.

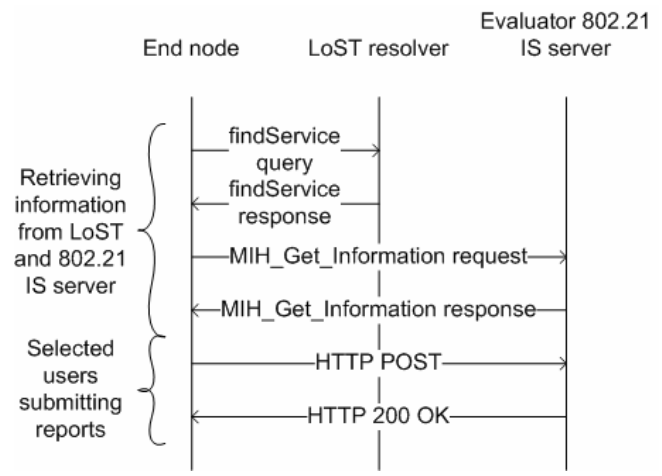

Fig. 6. Message exchange between end-users and Evaluator 802.21 IS servers 
Both LoST and IEEE 802.21 can handle provisioning of location-based services. Because of this, two architectural options are possible. One option is to have LoST provide coarse-grained information as well as fine-grained information. The first would be information regarding the available 802.21 IS servers to query, given the end-user's physical location while the latter would be about points of attachment the end-user could connect to. IEEE 802.21 IS servers would then take care only of QoSrelated information. In other words, reports about points of attachment would be sent to the LoST server and later queried via the LoST protocol, while reports about QoS information would be sent to IEEE 802.21 IS servers and later queried via the IEEE 802.21 MIH protocol.

In our proposed architecture, however, we follow a second option, which is to use LoST for the discovery of available 802.21 IS servers, given the end-user's current physical location, and then use 802.21 IS servers to deliver information to end-users about available points of attachment as well as QoS-related information. The reason for choosing this second approach is simply that we wanted to be consistent with the IEEE 802.21 framework without creating a special case based on LoST for the evaluator scope only, while having the other two scopes as IEEE 802.21 centric.

Furthermore, one issue with our proposal may be operators allowing access to their IEEE 802.21 IS servers only to closed user groups. Since subscribers most likely will be members of such user groups this is not a problem.

Finally, security and trust issues may occur when allowing users to contribute QoS-related information to Evaluator 802.21 IS servers. This aspect is, however, out of the scope of this paper.

\section{Experiments}

In order to evaluate our proposal, a prototype was built and tested in an experimental test-bed.

\subsection{Experimental Set-Up}

Our prototype was designed to demonstrate the key features of the proposed architecture in an experimental environment. The mobile node was implemented on a laptop running Linux Fedora core 12. Code for queries to the LoST system and the IEEE 802.21 IS servers was integrated with a commercial GPS system from Globalsat (BU353). For the LoST client/server we used the Columbia University LoST reference implementation [21]. An experimental 802.21 IS server was implemented using Python version 3.0 where Python classes for each 802.21 IE type were implemented and installed on a Linux server running Linux Fedora version 12. MIH protocol messages were transported over TCP using binary data transmission and TLV representation of IEs. The experimental 802.21 IS server was extended with web server capabilities for the handling of user-contributed reports on access networks over HTTP. Access network selection in the mobile node was performed by reusing principles from an earlier prototype [22] calculating policy values for each access technology taking the weighted sum of normalized values for cost, performance, and battery consumption into account. The access technology with lowest policy value was selected at each decision time. 
First, a trusted user follows a certain path and contributes with reports to the evaluator-operated IEEE 802.21 IS server. It provides information regarding the available points of attachment on that path. Sometime later, a regular user follows the same path and connects to those points of attachment discovered using the information provided by the evaluator-operated IEEE 802.21 IS server. Figures 7 a- $f$ indicate the path followed by such an end-user and the points of attachment provided by the IEEE 802.21 IS server on the path. Each time the user performs a query to the IEEE 802.21 IS server it specifies a circular area within which to search for points of attachment. Once the end-user reaches the limit of such circular area, new queries are issued.

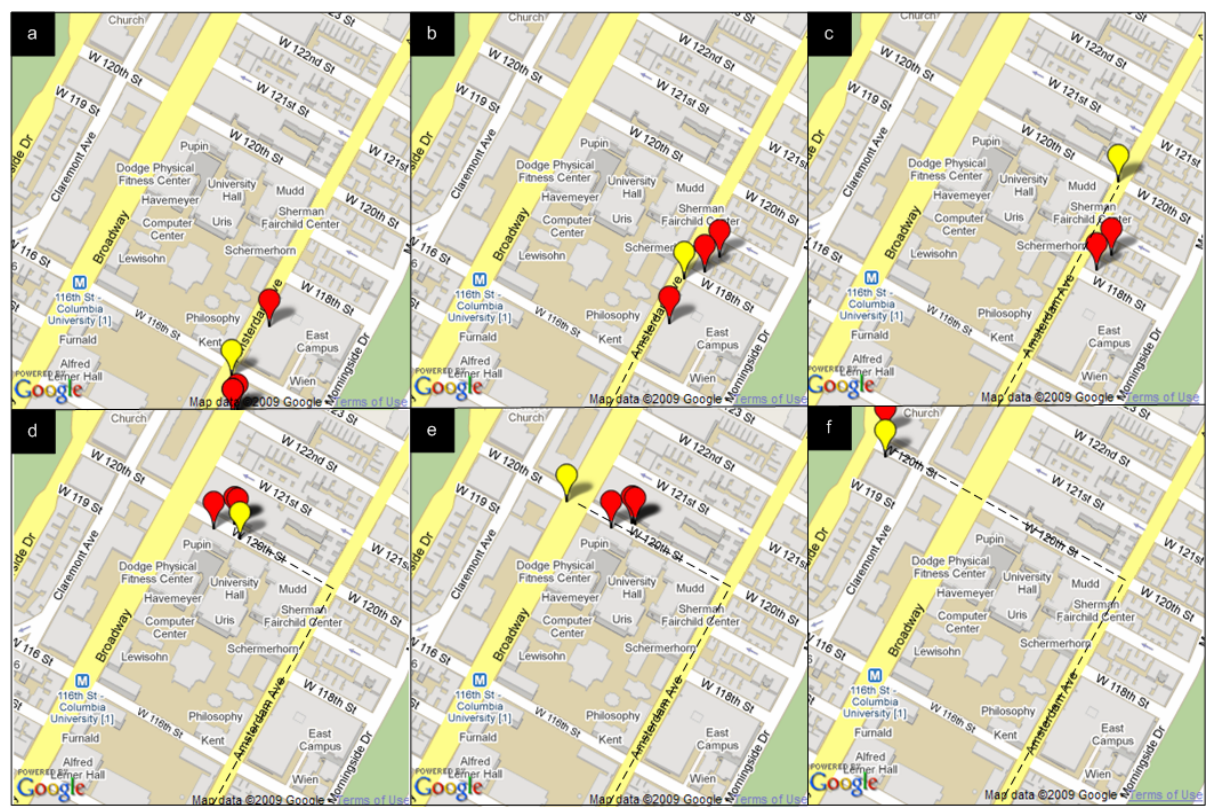

Fig. 7. a-f. Path followed by the end-user. The position of the end-user and points of attachment provided by the 802.21 IS server are indicated by different markers.

\subsection{Experimental Results}

Applying a make-before-break IP mobility management scheme with a multihomed mobile node as described in [22] avoided handoff delays and packet losses. Access to the evaluator-owned IEEE 802.21 IS server typically took an average of $22 \mathrm{~ms}$ for multiple-location queries. Such queries provide information on all the points of attachment present along the entire path. This is possible if the user already knows the entire path he or she will follow. Single location queries took an average of $19 \mathrm{~ms}$ and were performed so that new queries for points of attachment within 150 meters from the user's current location were made each 100 meters along the path. 


\section{Related Work}

To the best of our knowledge, the idea of helping roaming users by the delivery of IEEE 802.21 information services from independent evaluators is new. Also, the idea of looking up IEEE 802.21 IS servers through the LoST protocol is new. In the following we present some related work on IEEE 802.21 assisted handovers.

Eastwood et al. [23] showed how the requirements in the IMT-Advanced network (a.k.a. $4 \mathrm{G}$ ) can be met by integrating $802.11 \mathrm{VHT}$ and $802.16 \mathrm{~m}$ radio access technologies with the IEEE 802.21 handover support technology. Five use cases were identified and MIIS was used explicitly in the case where the user was decelerating while running an application whose performance was increasing at higher data rates.

Kim et al. [24] proposed a framework for seamless mobility across WIBRO and HSDPA networks using the IEEE 802.21 standard. The proposal was evaluated running Skype and a Video on Demand application in a multi-radio environment with a make-before-break handover policy. Results from a stationary test reported neither packet losses, nor any disruption of either voice or video streams. When driving at 60 $\mathrm{km} / \mathrm{h}$ service disruption occurred during handover in specific places, but overall performance was reported to be acceptable with no service quality degradation in the general case. The MIIS database was updated with data from users detecting a base station not yet included. This particular detail shows some similarity to the principles of our proposal. On the other hand, the approach of Kim et al. is to use a networkcentric access network selection mechanism which is not the case in our proposal.

Finally, there are quite a few papers where the IEEE 802.21 event and command services are used to improve handover performance. Cacace et al. [25] introduced the concept of a Mobility Manager (MM) interfacing with the MIH function in the mobile node. The MM included functionality for user policies, link quality, handoff decisions, and power management. By implementing a prototype on a Linux platform the proposal was tested in a UMTS/802.11 network environment. Adaptive streaming was also adopted, so that the bit-rates of the streams were adapted to the characteristics of the access technology being used for the moment. Also, adaptive VoIP applications were tested in the same manner. Results showed that connection and application quality improved.

Dutta et al. [26] described an experimental testbed including IEEE 802.21 features for network discovery and network selection. Experiments were performed with a VoIP application using a SIP-based mobility mechanism. Total handover delay was reported to $14 \mathrm{~ms}$ and only one single audio packet was lost at each handover.

On the commercial side, a number of web based Wi-Fi finder applications exist, but non-web-page versions being automated are not common. However, special applications for high end handsets like the Apple iPhone and its competitors exist [27]. The degree of automation in such applications varies a lot and standards-based solutions, like ours building on IEEE 802.21 and LoST, are not available.

Our approach combining the strengths of the LoST protocol and the IEEE 802.21 standard, and also allowing for independent evaluators to deliver information of interest when taking handover decisions makes it a very practical and useful solution to large groups of end-users. 


\section{Conclusions and Future Work}

The architecture proposed in this paper was described and evaluated through a prototype implementation and experiments in a live environment. It is a generic solution, allows for competition at all levels, and scales well due to the distributed nature of the proposed architecture. End-users will ultimately experience improved quality and lower costs. Also, as a consequence of basing the proposal on the IEEE 802.21 standard, the need for scanning has been minimized thus allowing for lower battery consumption.

Future work will include investigations on integrating the ideas presented in this paper with software defined radio (SDR) that is, solutions aiming to deliver "cellular on demand" services to end-users.

\section{Acknowledgement}

This work was supported in part by the National Science Foundation under award 0751094 and in part by Skellefteå Kraft within the Hybrinet project. The collaboration between Luleå University of Technology and Columbia University was financially supported by Nordea Norrland Foundation.

\section{References}

1. Perkins, C. (ed.): IP Mobility Support for IPv4, IETF, RFC 3344 (August 2002)

2. Johnson, D., Perkins, C., Arkko, J.: IP Mobility Support in IPv6, IETF, RFC 3775 (June 2004)

3. Stewart, R., Xie, Q., Morneault, K., Sharp, C., Schwarzbauer, H., Taylor, T., Rytina, I., Kalla, M., Zhang, L., Paxson, V.: Stream Control Transmission Protocol, IETF, RFC 2960 (October 2000)

4. Koh, S.J., Chang, M.J., Lee, M.: mSCTP for Soft Handover in Transport Layer. IEEE Communications Letters 8(3), 189-191 (2004)

5. Schulzrinne, H., Wedlund, E.: Application-layer mobility using SIP. Mobile Computing and Communications Review archive 4(3), 47-57 (2000)

6. Moskowitz, R., Nikander, P.: Host Identity Protocol (HIP) Architecture, IETF, RFC 4423 (May 2006)

7. Gundavelli, S. (ed.): Proxy Mobile IPv6, IETF, RFC 5213 (August 2008)

8. IEEE Standard for Local and metropolitan area networks-Part 21: Media Independent Handover Services (January 2009)

9. SPARQL Query Language for RDF, W3C Recommendation (January 2008), http: / / www.w3 .org/TR/2008/REC-rdf-sparql-query-20080115/

10. Melia, T. (ed.): Mobility Services Transport: Problem Statement, IETF, RFC 5164 (March 2008)

11. Melia, T.(ed.), Bajko, G., Das, S., Golmie, N., Zuniga, J.: IEEE 802.21 Mobility Services Framework Design (MSFD), IETF, RFC 5677 (December 2009)

12. Bajko, G., Das, S.: Dynamic Host Configuration Protocol (DHCPv4 and DHCPv6) Options for IEEE 802.21 Mobility Services (MoS) Discovery, IETF, RFC 5678 (December 2009)

13. Bajko, G.: Locating IEEE 802.21 Mobility Services Using DNS, IETF, RFC 5679 
14. Hardie, T., Newton, A., Schulzrinne, H., Tschofenig, H.: LoST: A Location-to-Service Translation Protocol, IETF, RFC 5222 (August 2008)

15. Schulzrinne, H.: Location-to-URL Mapping Architecture and Framework, IETF, RFC 5582 (September 2009)

16. Schulzrinne, H., Polk, J., Tschofenig, H.: Discovering Location-to-Service Translation (LoST) Servers Using the Dynamic Host Configuration Protocol (DHCP), IETF, RFC 5223 (August 2008)

17. Forte, A., Schulzrinne, H.: Location-to-Service Translation Protocol (LoST) Extensions, Internet Draft, draft-forte-ecrit-lost-extensions-02.txt (March 2009)

18. Forte, A., Schulzrinne, H.: Labels for Common Location-based Services, Internet Draft, draft-forte-ecrit-service-classification-02.txt (March 2009)

19. Forte, A., Schulzrinne, H.: Policy for defining new service-identifying labels, Internet Draft, draft-forte-ecrit-service-urn-policy-00.txt (March 2009)

20. Taniuchi, K., Ohba, Y., Das, S.: IEEE 802.21 Basic Schema, Internet Draft, draft-ohba802dot21-basic-schema-07.txt (December 2009)

21. Columbia University LoST Reference Implementation, http: //lost.cs.columbia.edu

22. Andersson, K., Granlund, D., Åhlund, C.: $\mathrm{M}^{4}$ : MultiMedia Mobility Manager - a seamless mobility management architecture supporting multimedia applications. In: Proceedings of the 6th International Conference on Mobile and Ubiquitous Multimedia, MUM2007, Oulu, Finland, December 2007. ACM International Conference Proceeding Series (2007)

23. Eastwood, L., Migaldi, S., Xie, Q., Gupta, V.: Mobility Using IEEE 802.21 In A Heterogeneous IEEE 802.16/802.11-based, IMT-Advanced (4G) Network. IEEE Wireless Communications 15(2), 26-34 (2008)

24. Kim, K., Hong, J.Y., Choi, Y., Kim, Y.H., Lee, S.: Design and Implementation of IEEE 802.21 Framework for WIBRO-HSDPA Seamless Mobility. In: Proceedings of 2009 International Conference on New Trends in Information and Service Science, Beijing, China (July 2009)

25. Cacace, F., Vollero, L.: Managing mobility and adaptation in upcoming 802.21 enabled devices. In: Proceedings of the 4th international Workshop on Wireless Mobile Applications and Services on WLAN Hotspots, Los Angeles, CA, USA (September 2006)

26. Dutta, A., Das, S., Famolari, D., Ohba, Y., Taniuchi, K., Kodama, T., Schulzrinne, H.: Seamless handover across heterogeneous networks - an IEEE 802.21 centric approach. In: Proceedings of the 8th International Symposium on Wireless Personal Multimedia Communications, Aalborg, Denmark (September 2005)

27. Wi-Fi Finder, http: //www.jiwire.com 